\title{
Computational methods and applications in queueing theory
}

\author{
Onno Boxma ${ }^{1} \cdot$ Joris Walraevens ${ }^{2}$
}

Published online: 16 March 2017

(C) Springer Science+Business Media New York 2017

This issue of the Annals of Operations Research is devoted to papers from the first European Conference on Queueing Theory (ECQT), which took place in Ghent, Belgium, August 2022, 2014. It continued a tradition started by the late Jesus Artalejo, who three times organized a Madrid Conference on Queueing Theory. ECQT featured about 80 presentations. The presenters of studies concerning computational methods and/or applications were offered the opportunity to submit a paper based on their presented work to Annals of Operations Research. The ten papers in this special issue have been chosen for publication after a careful refereeing process. We would like to take this opportunity to express our gratitude to Editorin-Chief Endre Boros, to Publications Manager Katie D'Agosta, and to the Springer staff for their advice, support, and help. We are also indebted to the TPC of ECQT for their aid in the review process and to the anonymous referees for their efforts. Below we give a brief overview of the accepted papers.

De Muynck, Wittevrongel, and Bruneel study a discrete-time queueing system where service capacities and service demands are explicitly modeled instead of being integrated in the traditional concept of service times. Both are modeled by means of i.i.d. random variables with general distributions. They derive (tail) distributions and moments for system occupancy and waiting time in this non-standard model. Rumyantsev and Morozov also study a queueing system with complications in the service times process. They model highperformance clusters by means of multiserver systems in which each customer requires a random number of servers simultaneously for a random amount of time. Stability criteria of such a system under exponential assumptions and with an arbitrary number of servers are obtained using a matrix-analytic approach. Another telecommunication application is studied

Joris Walraevens

Joris.Walraevens@UGent.be

Onno Boxma

o.j.boxma@tue.nl

1 Department of Mathematics and Computer Science, Eindhoven University of Technology, Eindhoven, The Netherlands

2 Department of Telecommunications and Information Processing, Ghent University, Ghent, Belgium 
by Kim and Hwang. They analyze the relation between delay performance and the selection distribution of the renewal access protocol, a simplified version of the IEEE 802.11 DCF protocol. With the help of effective bandwidth theory, they derive conditions for the selection distribution to optimize the queue overflow probability. However, they also show that it has slow convergence to steady state compared with that of a Poisson selection distribution.

Besides communication and computer networks, call centers and risk management are still hot application domains for queueing theory. Chen and Worthington study call centers with time-dependent arrivals requiring time-dependent staffing levels. They evaluate an analytical queueing model combined with an iterative staffing algorithm to be used for setting staffing levels to achieve time-stable performance in call center type queues. Results show that the method is faster than simulations while it is more accurate than standard analytical methods. Yazici and Akar present a new numerical method to obtain ruin probabilities for a general continuous-time risk problem with claims arriving according to a Markovian Arrival Process, phase-type claim sizes, and multi-threshold premiums. By means of a sample path technique, it is shown that the steady-state solution reduces to that of a certain multi-regime Markov fluid queue. Tschaikowski and Tribastone prove convergence of steady-state open queueing networks with many-server stations and Coxian-distributed service and abandonment times to a fluid limit. The fact that their coupled ordinary differential equations are piecewise affine enables a computational method for establishing the presence of a global attractor, based on the solution of a system of linear matrix inequalities.

Two papers in this special issue discuss new aspects of some traditional queueing models. Kim and Kim study a two-class priority M/G/1 retrial queueing system. Arriving high-priority customers queue in a regular infinite-capacity queue, while low-priority customers have to retry from a retrial orbit if the server is unavailable. Waiting time moments and distributions of both types of customers are analyzed. Chaudhry, Banik, and Pacheco analyze a batch arrival single-server queue with generally distributed batch interarrival times, generally distributed batch sizes, and a Markovian service process. The analysis is based on roots of the associated characteristic equation of the vector-generating function of system-length distribution at an arrival instant. They also establish heavy- and light-traffic approximations.

Finally, the last two papers concern the output and loss process respectively. Steyaert, Wittevrongel, and Bruneel characterize the output process of network nodes. They derive an analytic closed-form formula for the burst factor, a measure for the long-term variability of a packet stream, of the output of a discrete-time $G I / D / 1$ queueing system, in terms of parameters of the arrival process. Ferreira, Pacheco, and Ribeiro turn their attention to loss characteristics associated with busy periods of regular and non-preemptive oscillating $M^{X} / G / 1 / n$ queueing systems. A fast recursive procedure, that is also easy to implement, is proposed to compute integer moments of the number of customers lost in busy periods, by taking advantage of the Markov regenerative structure of the number of customers in the system. 